\title{
An Efficient Technique for Image Mosaicing using Random Sample Consensus Algorithm
}

\author{
Nikhil Ranjan \\ PG Scholar NRI Institute of \\ Information Science \& \\ Technology, Bhopal (M.P.) \\ India
}

\author{
Braj Bihari Soni \\ Asst. Professor NRI Institute of \\ Information Science \& \\ Technology, Bhopal (M.P.) \\ India
}

\author{
Brahmi Shraman \\ Asst. Professor NRI Institute of \\ Information Science \& \\ Technology, Bhopal (M.P.) \\ India
}

\begin{abstract}
Image mosaicing algorithm based on random corner method is proposed. An image mosaicing is a method of combining multiple photographic with overlapping fields of view to produce a segmented panorama of high resolution image. The output of image mosaic will be the combination of two input images. In this paper we are using three step image mosaic methods. The first step is taking two input images and finding out the corners in both the images, second step is removing out the false corner in both the images and then by using homography we find its matched corner pair and we get final output mosaic. The experimental results show the proposed algorithm produces an improvement in mosaic accuracy, efficiency and robustness.
\end{abstract}

Keywords: Blending, Homography, Image, Registration, Stitching, Warping.

\section{INTRODUCTION}

An image is an array, or a matrix, of square pixels (picture elements) arranged in columns and rows. An image is also defined as a two dimensional function $(\mathrm{x}, \mathrm{y})$, where $\mathrm{x}$ and $\mathrm{y}$ are spatial (plane) coordinates, and the amplitude of $f$ at any pair of coordinates $(x, y)$ is called the intensity or gray level of the image at that point. The mosaic as an art form is defined in the Merriam-Webster dictionary as "a surface decoration made by inlaying small pieces of variously coloured material to form pictures or patterns." The mosaic dates to the 4th century B.C., and is generally associated with the Greeks. In fact, the word mosaic is of Greek origin, meaning "patient work of art, worthy of the muses".

Image mosaicing is the process of combining multiple overlapping images of same scene into a larger image. The output of image mosaicing operation will be the combination of input images. Image mosaicing is an effective means of constructing a single panoramic image from a series of snapshots taken in different viewing angles. An Image mosaic is a synthetic composition generated from a sequence of images and it can be obtained by understanding geometric relationships between images. The geometric relations are coordinate transformations that relate the different image coordinate systems. By applying the appropriate transformations via a warping operation and merging the overlapping regions of warped images, it is possible to construct a single image indistinguishable from a single large image of the same object, covering the entire visible area of the scene.

Various steps in mosaicing are feature extraction and registration, stitching and blending. Image registration refers to the geometric alignment of a set of images. The set may consist of two or more digital images taken of a single scene at different times, from different sensors, or from different viewpoints. The goal of registration is to establish geometric correspondence between the images so that they may be transformed, compared, and analyzed in a common reference frame. This is of practical importance in many fields, including remote sensing, medical imaging, and computer vision [1]. Registration methods can be loosely divided into the following classes: algorithms that use image pixel values directly, e.g., correlation methods [2]; algorithms that use the frequency domain, e.g., fast Fourier transform based (FFT-based) methods [1] algorithms that use low-level features such as edges and corners, e.g., feature based methods [3]; and algorithms that use high-level features such as identified (parts of) objects, or relations between features, e.g., graph-theoretic methods.

The next step, following registration, is image stitching. Image integration or image stitching is a process of overlaying images together on a bigger canvas [4] \& [5]. Image Blending is the technique which modifies the image grey levels in the terms of a boundary to obtain a smooth transition between images by removing these seams and creating a blended image.

\section{DESIGN METHODOLOGY}

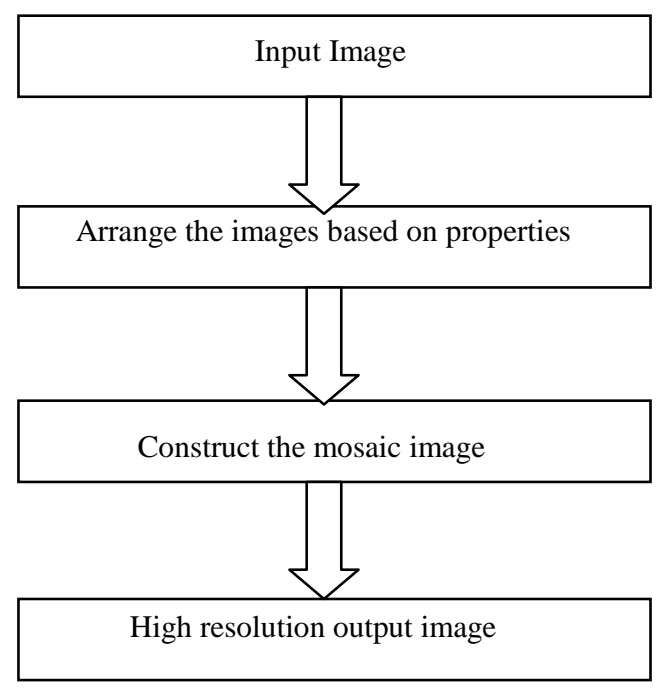

Fig 1: Existing framework for image mosaicing 


\section{FEATURE POINT EXTRACTION}

\subsection{Idea of Harris Corner}

The first step in the image mosaic process is feature detection The efficiency of extracted feature points in the two images is ascertained by the invariance and accuracy of the feature detector in the overlapping region. Therefore we introduce Harris detector in our mosaic framework. Harris corner detecting was point feature extracting algorithm by C. Harris and M.J. Stephens in 1988. Its main idea is to design a local detecting window in image. When the window moves in each direction the average grey variation of window is more than threshold, then the centre point of the window is extracted as corner point[6], [7] \& [8]. When we just shift one pixel in an image that can create a significant change in the corner.

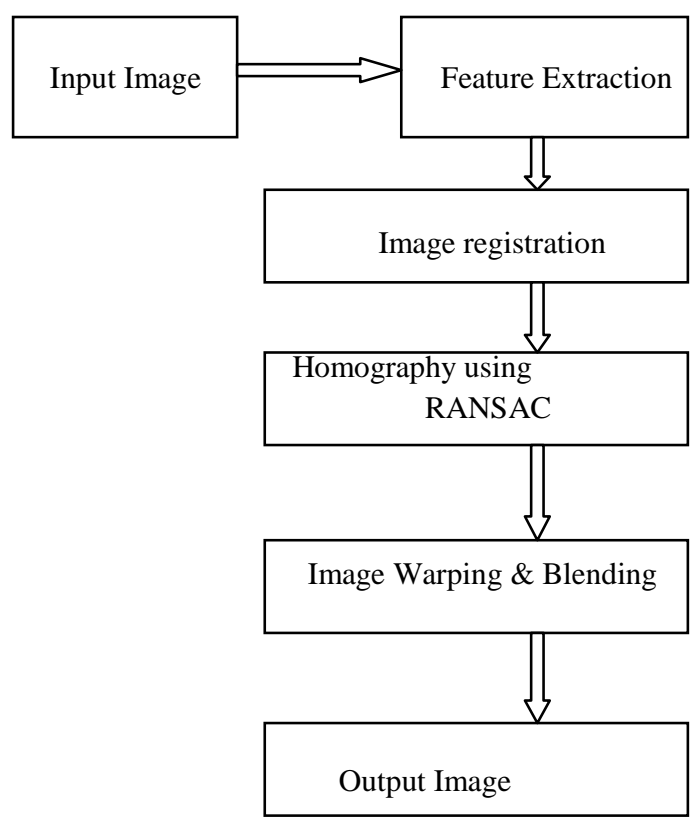

Fig 2: Proposed framework for image mosaicing

Considering gray intensity of pixel $(\mathrm{u}, \mathrm{v})$ be $\mathrm{I}(\mathrm{x}, \mathrm{y})$, the variation of gray pixel $(x, y)$ with a shift of $(u, v)$ can be denoted as

$\mathrm{E}(\mathrm{u}, \mathrm{v})=\sum_{x, y} w(x, y)[\mathrm{I}(\mathrm{x}+\mathrm{u}, \mathrm{y}+\mathrm{v})-\mathrm{I}(\mathrm{x}, \mathrm{y})]^{2}$

On applying Taylor series expansion we have

$\mathrm{E}(\mathrm{u}, \mathrm{v})=\sum_{x, y} w(x, y)\left[\mathrm{I}_{\mathrm{x}} \mathrm{u}+\mathrm{I}_{\mathrm{y}} \mathrm{v}+\mathrm{O}\left(\mathrm{u}^{2}, \mathrm{v}^{2}\right)\right]^{2}$

For a small shift of $(\mathrm{u}, \mathrm{v})$ we have

$$
\mathrm{E}(\mathrm{u}, \mathrm{v})=[\mathrm{u}, \mathrm{v}] \mathrm{M}\left[\begin{array}{l}
u \\
v
\end{array}\right]
$$

Where $\mathrm{M}$ is a matrix of $2 \mathrm{X} 2$ which has been calculated from the image derivatives:

$\mathrm{M}=\sum_{x, y} w(x, y)\left[\begin{array}{cc}I_{x}^{2} & I x I y \\ I x I y & I_{y}^{2}\end{array}\right]$

If $\lambda 1, \lambda 2$ are the Eigen values of matrix $M$, then corner, edge, and flat area of the image can be computed from the Eigen values as follows:
- $\quad$ Flat area: both $\lambda 1, \lambda 2$ are very small.

- Edge: one of $\lambda 1, \lambda 2$ is smaller and the other is bigger.

- Corner: both $\lambda 1, \lambda 2$ are bigger and are nearly equal.

For extracting the corner, Harris gives a formula

$$
\mathrm{R}=\operatorname{det} \mathrm{M}-(\text { trace } \mathrm{M})^{2}
$$

Where,

$$
\begin{aligned}
& \operatorname{det} M=\lambda 1 \quad \lambda 2 \\
& \text { trace } M=\lambda 1+\lambda 2
\end{aligned}
$$

\subsection{Algorithm Steps}

Input: Gray scale image, Gaussian variance, k Value, Threshold $\mathrm{T}$

Output: Map indicating position of each detected corner.

1 for each pixel $(x, y)$ in image calculate the Autocorrelation matrix M:

$$
\mathrm{M}=\sum_{x, y} \quad\left[\begin{array}{cc}
I_{x}^{2} & I x I y \\
I x I y & I_{y}^{2}
\end{array}\right]
$$

Where,

2 for each pixel of image has Gaussian filtering, get new matrix $\mathrm{M}$, and discrete two dimensional zero-mean Gaussian function as follows:

$$
\text { Gauss }=\exp \left[-\frac{\left(u^{2}+v^{2}\right)}{2 \delta^{2}}\right]
$$

3 Calculating the cornerness measure for each pixel $(x, y)$, get $\mathrm{R}$ :

$$
\mathrm{R}=\left[\mathrm{I}_{\mathrm{x}}{ }^{2} \mathrm{xI} \mathrm{I}_{\mathrm{y}}{ }^{2}-\left(\mathrm{I}_{\mathrm{x}} \mathrm{I}_{\mathrm{y}}\right)^{2}\right]-\mathrm{K}\left[\mathrm{I}_{\mathrm{x}}{ }^{2} \mathrm{I}_{\mathrm{y}}{ }^{2}\right]^{2}
$$

4 Choose the local maximum point, Harris method consider that the feature points are the pixel value which corresponding with the local maximum interest point.

5 Set the threshold T, detect corner points

\section{IMPROVED ALGORITHM FOR HARRIS CORNER}

In this novel we adopt neighbouring point eliminate method to avoid setting threshold $\mathrm{T}$, ensure the corner will homogeneous distribution and avoid corner clustering. We can select one stencil (e.g. 3X3) to process for image. If there is not only one corner point under this stencil, we retain the corner which has the largest value of $\mathrm{R}$. Experimental shows that the method inhibition the clustering phenomenon better.

Firstly detect all corner points $\mathrm{C}$ (i, j) in the image, and calculate $\mathrm{R}$ save them into matrix Mat [Sum], Sum is the number of the corners. Then sort new sequence by the value of $\mathrm{R}$, and save them into the matrix Mat1 [Sum], choose the large value of $R$ in the matrix Mat1 [Sum] for the final detection corner. At last we have the neighbour point eliminate processing for image. If there are more than one corner point, retain the corner point which has the large value of $\mathrm{R}$.

\section{HOMOGRAPHY COMPUTATION}

\subsection{Homography}

It is mapping between two spaces which is often used to represent the correspondence between two images of the same scene. It is widely used for the project where multiple images are 
taken from a rotating camera center ultimately warped together to produce a panoramic view

The steps for homography detection algorithm using RANdom Sample Consensus (RANSAC)[9] scheme is

1. Firstly, corners are detected in both images.

2. Variance normalized correlation is applied between corners, and pairs with a sufficiently high correlation score are collected to form a set of candidate matches.

3. Four points are selected from the set of candidate matches, and a homography is computed.

4. Pairs agreeing with the homography are selected. A pair (p, $\mathrm{q}$ ), is considered to agree with a homography $\mathrm{H}$, if for some threshold: $\operatorname{Dist}(\mathrm{Hp}, \mathrm{q})<€$

5. Steps 3 and 4 are repeated until a sufficient number of pairs are consistent with the computed homography.

6. Using all consistent correspondences, the homography is recomputed by solving step 4 .

\subsection{RANSAC Algorithm}

RANSAC, is a method to calculate the parameters of a mathematical model from a set of observed data.. Input of RANSAC algorithm is a set of observed data, a parameterized model which can explain or fit to the observations, along with some confidence parameters. RANSAC achieves its goal by iterative selection of a random subset of the original data

Given a fitting problem with parameter $x$, it estimates the parameters considering the following assumption:

1. Parameter can be estimated from $\mathrm{N}$ data items.

2. Available data items are totally $M$

3. The probability of a randomly selected data item being part of a good model is $\mathrm{Pg}$

4. The probability that the algorithm exit without finding a good fit if one exists is Pf

Then the algorithm is:

1. $\mathrm{N}$ data items at random are selected.

2. Parameter $\mathrm{x}$ is estimated.

3. Number of data items which fit the model with parameter vector $\mathrm{x}$ are found out within a user defined tolerance. Let it be $\mathrm{K}$. 4. If $\mathrm{K}$ is large enough, it is accepted and exit with success.

5. The process is repeated $1.4 \mathrm{~L}$ times

6. The process is failure if it again enters the loop.

Value of $\mathrm{L}$ is found by the following formulae:

$P_{\text {fail }}=$ Probability of $\mathrm{L}$ consecutive failures

$=$ Probability that a given trial is a failure) $\mathrm{L}$

$=(1-($ Probability that a random data item fits the model $) \mathrm{N}) \mathrm{L}$

$$
\begin{aligned}
& \mathrm{P}_{\text {fail }}=\left[1-\left(\mathrm{P}_{\mathrm{g}}\right)^{\mathrm{N}}\right]^{\mathrm{L}} \\
& L=\frac{\log \left(P_{\text {fail }}\right)}{\log \left(1-\left(P_{g}\right)^{N}\right)}
\end{aligned}
$$

\section{IMAGE WARPING \& BLENDING}

\subsection{Image warping}

Image warping is the process of digitally employing an image such that any shapes represented in the image have been significantly distorted. Warping can also be used for correcting image distortion as well as for inventive purposes. Basically we can simply warp all the input images to a plane defined by one of them known as reference image. The two images that will form the mosaic are warped, by using the geometric transformation. While an image can be reconstituted in various ways, pure warping means that points are mapped to points without changing the colors. It can be mathematically based on any function from the plane to the plane. If the function is injective then the original can be reconstructed.

\subsection{Image Blending}

The final step is to blend the pixel colors in the overlapped region to avoid the seams. Simplest available form is to use feathering, which uses weighted averaging color values to blend the overlapping pixels. We generally use alpha factor often called alpha channel having the value 1 at the center pixel and becomes 0 after decreasing linearly to the border pixels. Where at least two image overlap occurs in an output mosaic we will use the alpha values as follows to compute the color at a pixel in there, suppose there are 2 images, $I 1, I 2$, overlapping in the output image; each pixel $(x, y)$ in image $I_{\mathrm{i}}$ is represented as $I_{i}(x$, $y)=(\alpha i \mathrm{R}, \alpha \mathrm{iG}, \alpha \mathrm{iB}, \alpha \mathrm{j})$ where $(\mathrm{R}, \mathrm{G}, \mathrm{B})$ are the color values at the pixel. We will compute the pixel value of $(x, y)$ in the stitched output image as $[(\alpha 1 \mathrm{R}, \alpha 1 \mathrm{G}, \alpha 1 \mathrm{~B}, \alpha 1)+(\alpha 2 \mathrm{R}, \alpha 2 \mathrm{G}$, $\alpha 2 \mathrm{~B}, \alpha 2)] /(\alpha 1+\alpha 2)$.

\section{EXPERIMENT}

The algorithm proposed here has been implemented in Matlab R2010a and has been executed in system with configuration i5 processor, 4 GB RAM, 2 GB cache memory and $2.8 \mathrm{GHz}$ processor. Fig 3 is the original input images and it provides the corners by using Harris corner detector. Fig 4 show the result of corner by using improved Harris corner Detector. Fig 5 shows the rough match result by normalized cross correlation method. Fig 6 removing the false matched corner. Fig 7 and 8 shows the matched corner points in both the input images by using RANSAC algorithm after the output image mosaic is obtained.

Table 1 represents a qualitative analysis of an existing algorithm [10] and the proposed algorithm for image blending. PSNR and entropy is increasing which shows the better quality of mosaic image. However, the value of blur metric is decreasing that shows the smoothing of the image.

Table 1: Qualitative Assessment

\begin{tabular}{|c|c|c|c|}
\hline \multirow{2}{*}{$\begin{array}{c}\text { Blending } \\
\text { Algorithms }\end{array}$} & \multicolumn{3}{|c|}{ Quality assessment parameters } \\
\cline { 2 - 4 } & PSNR(dB) & Entropy & Blur Metric \\
\hline Existing Algorithm & 28.6264 & 3.6672 & 0.1825 \\
\hline Proposed Algorithm & 28.6657 & 7.5613 & 0.1674 \\
\hline
\end{tabular}

\section{RESULTS}

We are comparing the original algorithm and the improved algorithm. Fig 3 is two groups of the original images. 


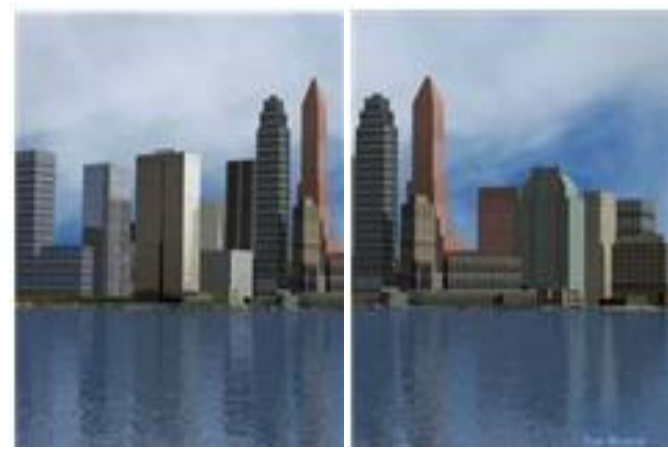

Fig 3: The original input images

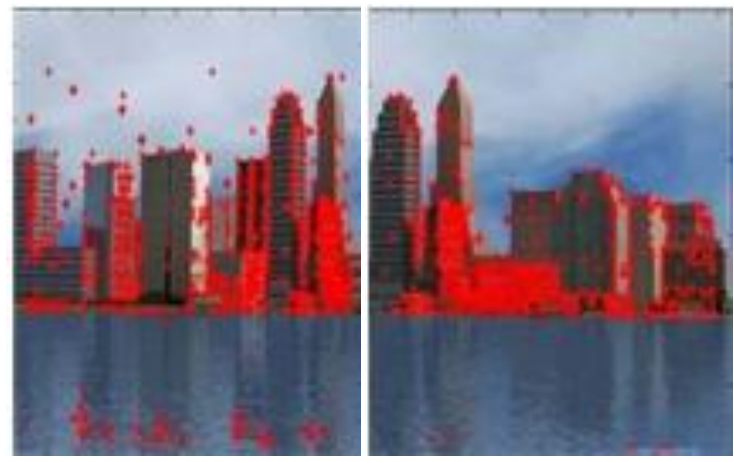

Fig 3: Original algorithm detect corners

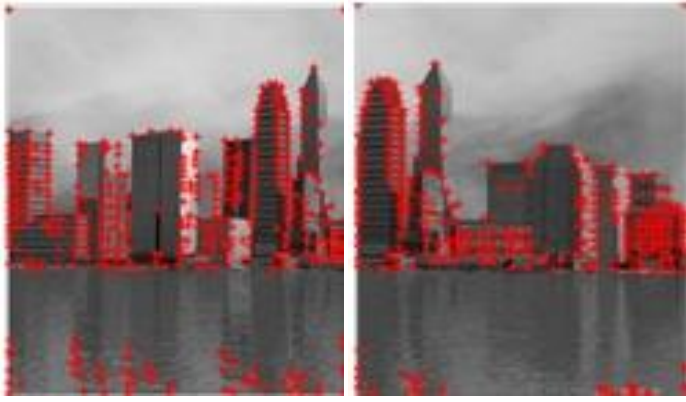

Fig 4: Improved algorithm detect corners

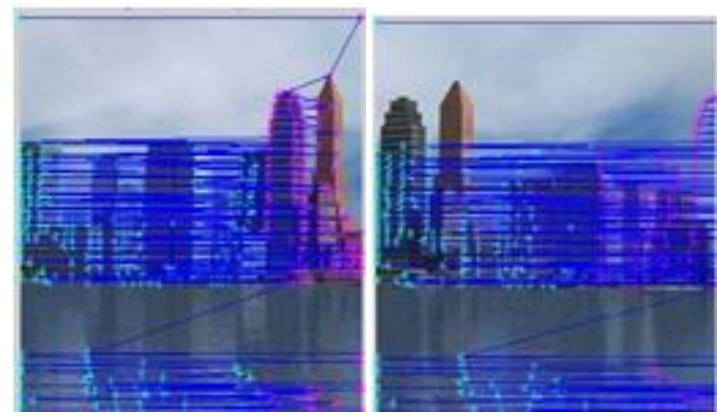

Fig 5: Rough match result by NCC
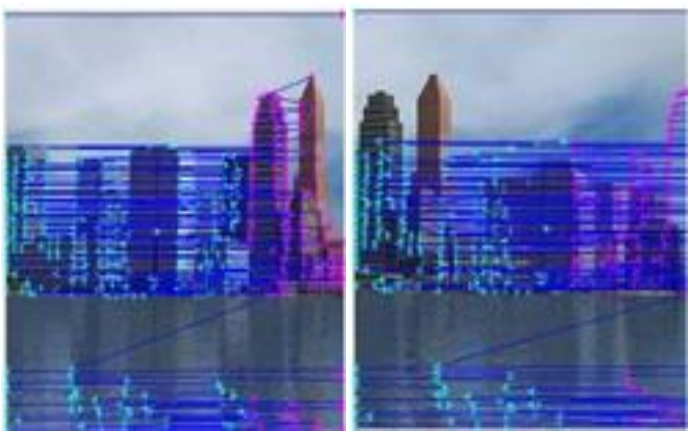

Fig 6: Remove the false matched pair

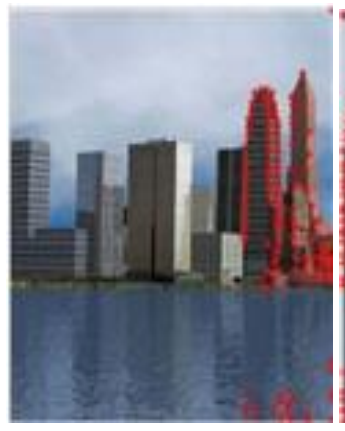

Fig 7: Matched points of image 1

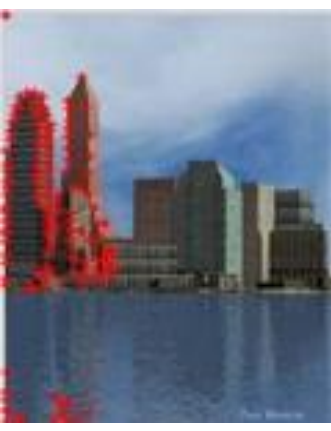

Fig 8: Matched points of image 2

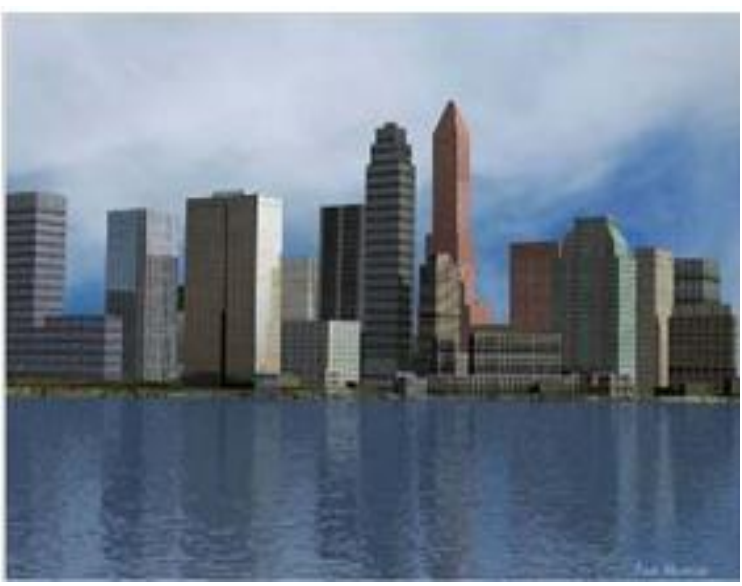

Fig 9: Result of image mosaic

\section{CONCLUSION}

The proposed method applies the corner detector technique to extract feature points from a partially overlapping image pair. By defining a similarity measure metric, the two sets of feature points can be compared, and the correspondences between the feature points can be established by using Normalized Cross Correlation Method. Once the set of correctly matched feature point pairs between two images are found, the registration parameters can be derived accordingly. Hence the registered image of two input images can be obtained. Finally we got Seamless Mosaic Image. 
In the future, we can implement image mosaicing algorithm on video sequences registration method. The idea of an ultimate registration method, able to recognize the type of given task and to decide by itself about the most appropriate solution, can motivate the development of expert systems. They will be based on the combination of various approaches, looking for consensus of particular results.

\section{REFERENCES}

[1] C. D. Kuglin and D.C. Hines "The phase correlation image alignment method," in Proc. 1975 Int. Conf. Cybernet Society, New York, NY, pp. 163-165

[2] D. I. Barnea and H.F. Silveman, "A class of algorithm for fast digital registration,"IEEE Transaction, Comput, vol. c21, pp. 179-186, 1972.

[3] Lisa G, Brown, "A survey of image registration techniques." ACM Computing Surveys, 24(4):325-376, December 1992.

[4] Xiong Shi, Jun Chen "Image moaic algorithm based on feature block matching," IEEE Tranc. 2011.
[5] Chao Sui, Ngai Ming Kwok, T. Ren "Image mosaic construction using feature matching and weighted fusion," IEEE, 2010.

[6] Wan, Yanli Miao, Zhenjiang Chen, Jing, "A Robust Image Mosaic Algorithm", Image and Signal Processing, 2008 Congress, 766 - 770, May 2008.

[7] Gao Guandong Jia Kebin, "A New Image Mosaics Algorithm Based on Feature Points Matching", Second International Conference on Innovative Computing, Information and Control, 2007. ICICIC '07, 471 - 471, Sept. 2012.

[8] D. Marr and E. Hildreth, "Theory of Edge Detection," Proc. of Royal Soc. London B ,vol. 207, pp. 187-217, 1980.

[9] Fischler, M. A. And Bolles, R.C., "Random sample consensus: a paradigm for model fitting with applications to image analysis and automated cartography", Communication Association Machine, 1981, pp. 381-395.

[10] Achala Pandey, Umesh C. Pati, "A novel technique for Non-Overlapping image mosaicing based on pyramid method" IEEE, 2013. 